\title{
Vor den Koalitionsverhandlungen: Bibliotheken drängen auf mehr politische Unterstützung und bessere Rahmenbedingungen in neuer Legislaturperiode
}

https://doi.org/10.1515/bd-2022-0004

Anlässlich der intensivierten Sondierungsgespräche zwischen SPD, Bündnis 90/ Die Grünen und FDP erinnert der Deutsche Bibliotheksverband e.V. (dbv) an seine wichtigsten bibliothekspolitischen Anliegen auf Bundesebene:

- Einbeziehung von außerschulischen Bildungsakteuren wie Bibliotheken in die Strategien und Initiativen zur (digitalen) Bildung;

- Finanzielle Unterstützung für die weitere digitale Transformation von Bibliotheken sowie die Digitalisierung des Kulturerbes. Schaffung einer international koordinierenden Infrastruktur zur Vermeidung von Doppeldigitalisierungen sowie Unterstützung der nationalen Allianz zur Kulturgutdigitalisierung;

- Unterstützung und finanzielle Förderung des Ausbaus von Bibliotheken aller Größenordnungen als „Begegnungsorte“;

- Sicherstellung, dass Bibliotheken beim E-Lending aus allen auf dem Markt erhältlichen Werke auswählen und auch Neuerscheinungen zu angemessenen Bedingungen an ihre Nutzer^innen verleihen können;

- Schaffung eines zeitgemäßen und dem digitalen Wandel angepassten Urheberrechts. Insbesondere: Erleichterung der zeithistorischen Forschung durch Erweiterung der Möglichkeit des Kopienversands durch Bibliotheken auf Zeitungen und Publikumszeitschriften;

- Verstetigung der Koordinierungsstelle für die Erhaltung des schriftlichen Kulturguts (KEK) und Erhöhung ihrer jährlich zur Verfügung stehenden Fördermittel;

- Ausweitung der Möglichkeit der Sonntagsöffnung im Bundesarbeitszeitgesetz auf kommunale Bibliotheken - in Verbindung mit einer angemessenen finanziellen Unterstützung und Personalausstattung.

$\mathrm{Zu}$ den Forderungen sagt der Bundesvorsitzende des dbv, Prof. Dr. Andreas Degkwitz: „Als Orte des Austauschs und des Miteinanders, der Information und der Medien sowie der (digitalen) Bildung leisten Bibliotheken einen unverzichtbaren Beitrag zu unserer Gesellschaft. Dafür müssen sie weitere politische Unter- 
stützung für ihre Vorhaben erhalten, und es müssen die rechtlichen und finanziellen Rahmenbedingungen stimmen. Die möglichen Koalitionspartner haben jetzt die Chance, die bibliothekspolitischen Anliegen frühzeitig aufzunehmen und in der neuen Legislatur aktiv anzugehen.“

\section{Deutscher Bibliotheksverband}

Der Deutsche Bibliotheksverband e.V. (dbv) vertritt mit seinen fast 2.100 Mitgliedern bundesweit über 9.000 Bibliotheken mit 25.000 Beschäftigten und 11 Millionen Nutzer^innen. Sein zentrales Anliegen ist es, Bibliotheken zu stärken, damit sie allen Bürger`innen freien Zugang zu Informationen ermöglichen. Der Verband setzt sich ein für die Entwicklung innovativer Bibliotheksleistungen für Wissenschaft und Gesellschaft. Als politische Interessensvertretung unterstützt der dbv die Bibliotheken insbesondere auf den Feldern Informationskompetenz und Medienbildung, Leseförderung und bei der Ermöglichung kultureller und gesellschaftlicher Teilhabe für alle Bürger^innen. URL: www.bibliotheksverband.de

\section{Pressekontakt}

Kristin Bäßler

Deutscher Bibliotheksverband

Leitung Kommunikation / Pressesprecherin

Tel.: + 49 (0)30 6449899 25 | E-Mail: baessler@bibliotheksverband.de 